\title{
Calibration and evaluation of CCD spectroradiometers for ground-based and airborne measurements of spectral actinic flux densities
}

Birger Bohn and Insa Lohse

Correspondence to: Birger Bohn (b.bohn@fz-juelich.de)

The copyright of individual parts of the supplement might differ from the CC BY 3.0 License. 

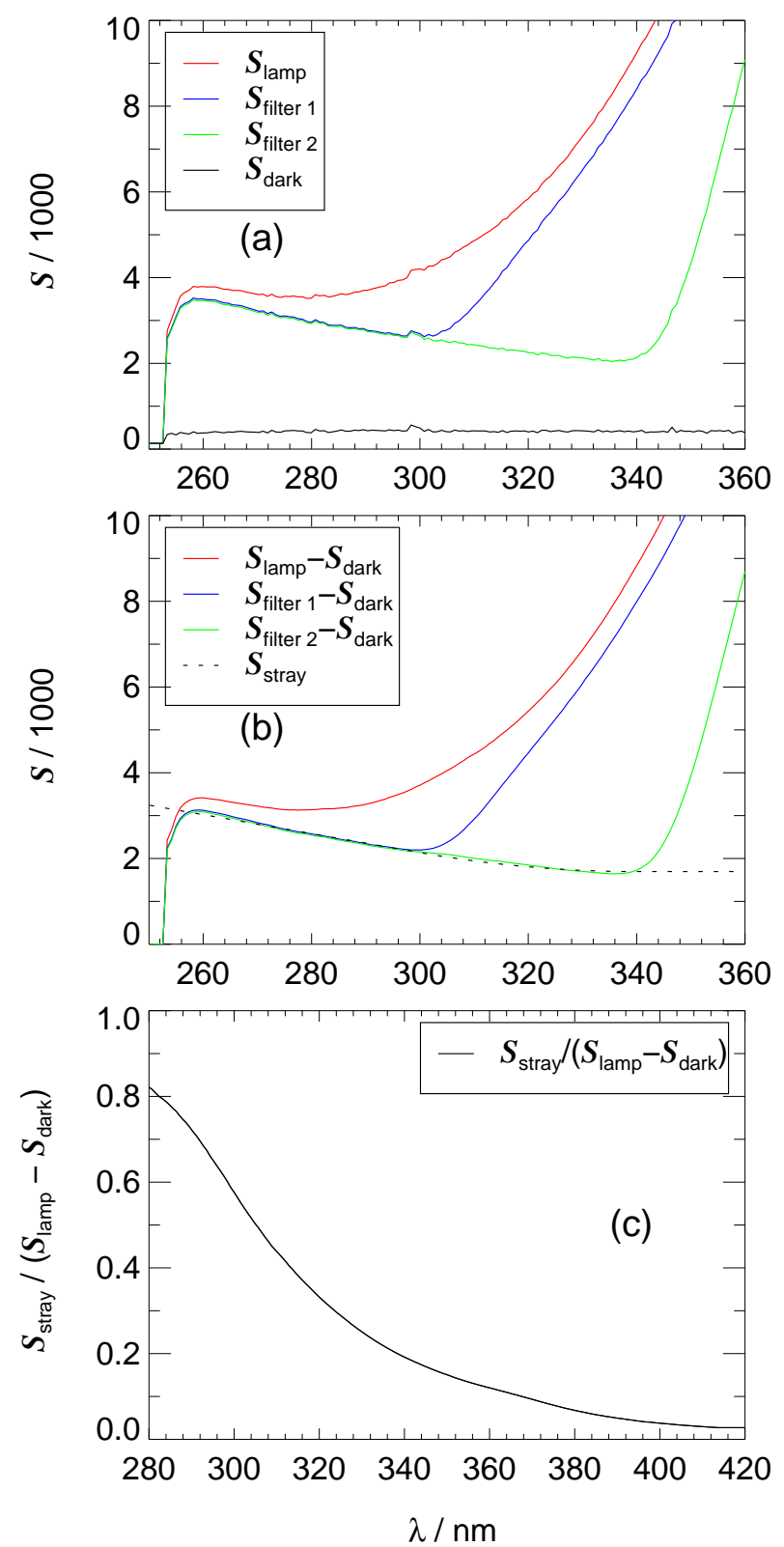

Figure S1: Example signals obtained during laboratory calibration measurements of instrument 45853 with 1000 ms integration time and two cutoff filters. Refer to Sect. 2.2.3 and caption of Fig. 6 for more details.
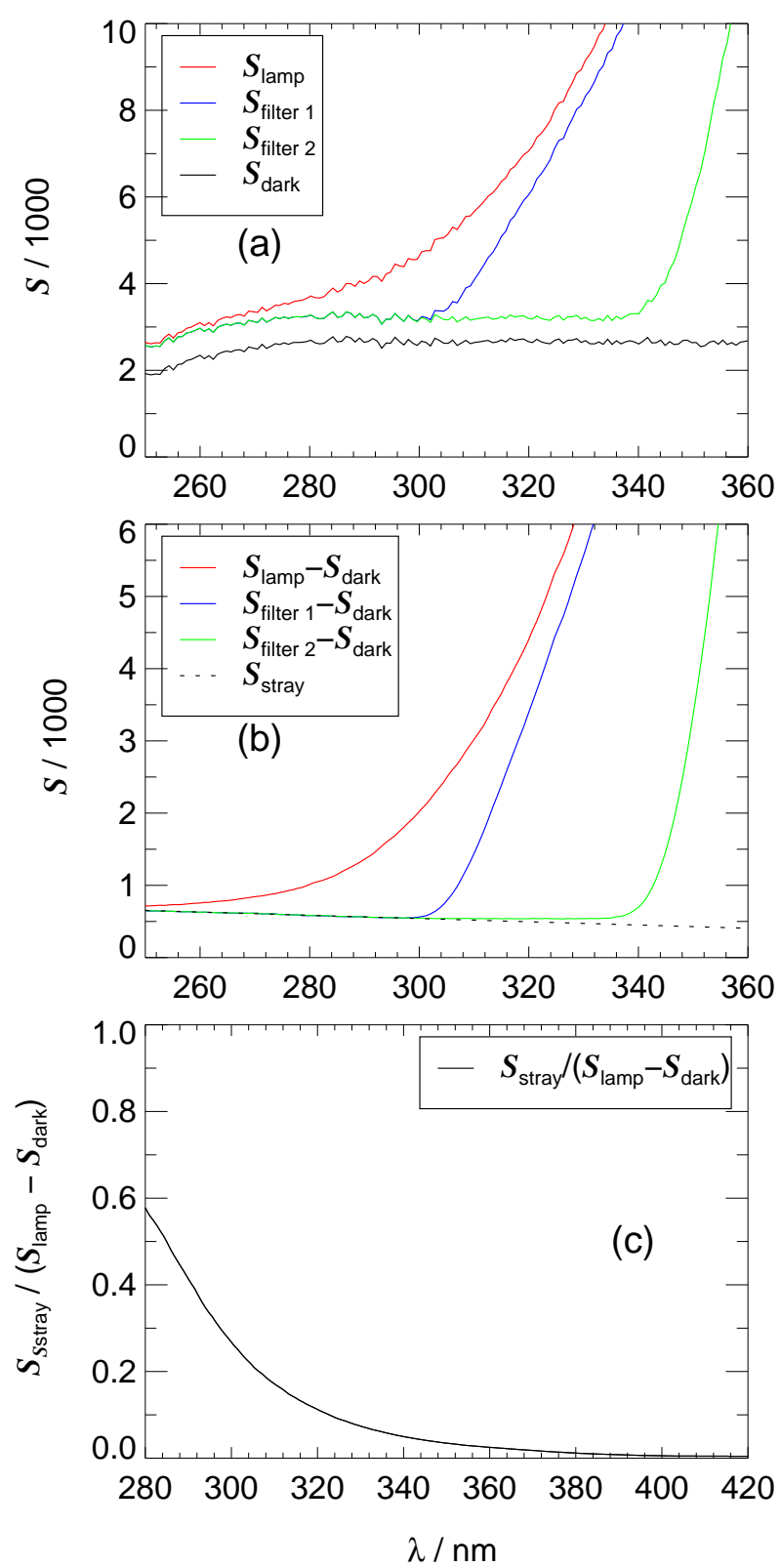

Figure S2: Example signals obtained during laboratory calibration measurements of instrument 62000 with $1000 \mathrm{~ms}$ integration time and two cutoff filters. Refer to Sect. 2.2.3 and caption of Fig. 6 for more details. 

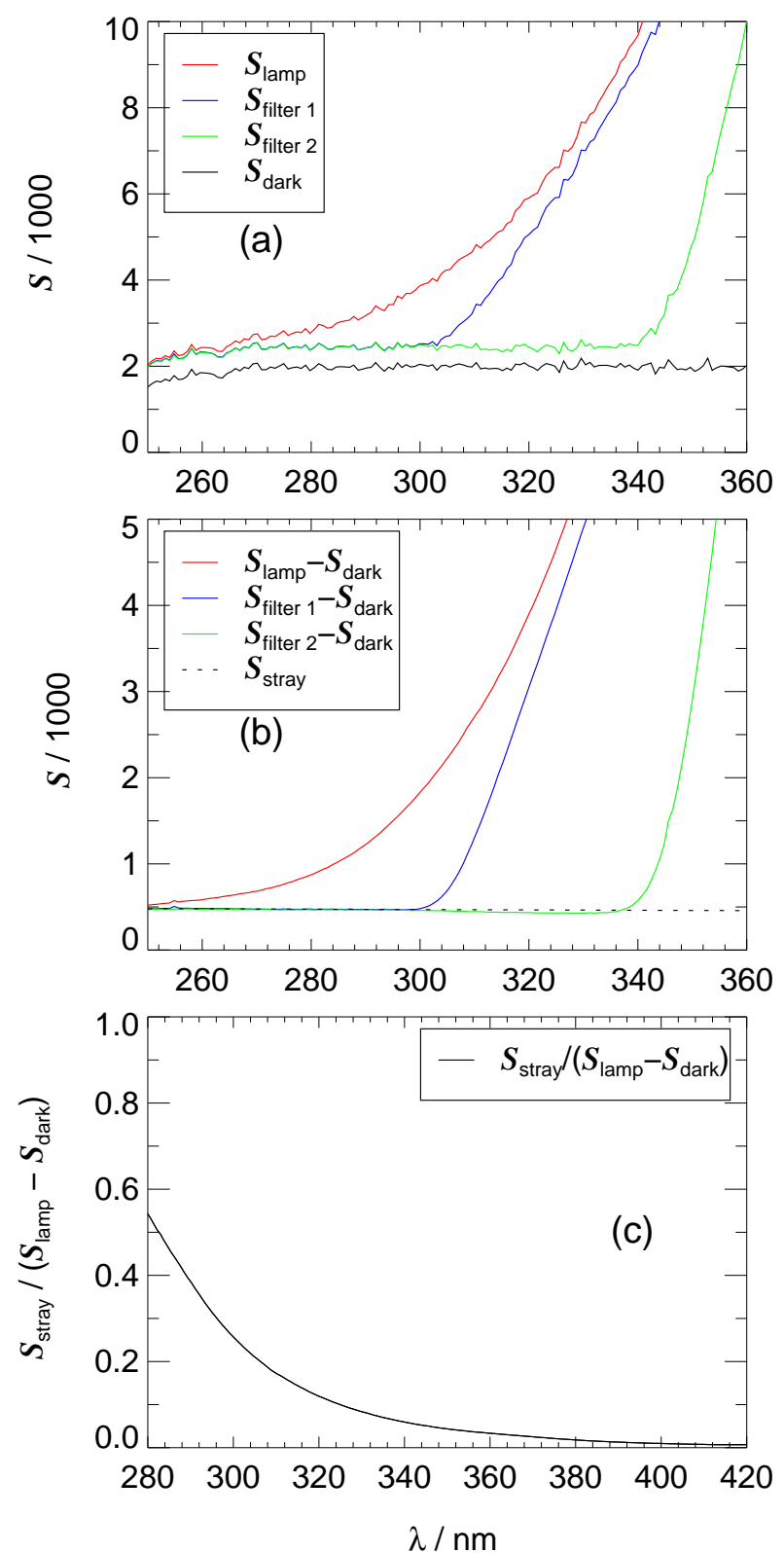

Figure S3: Example signals obtained during laboratory calibration measurements of instrument 62008 with 1000 ms integration time and two cutoff filters. Refer to Sect. 2.2.3 and caption of Fig. 6 for more details.
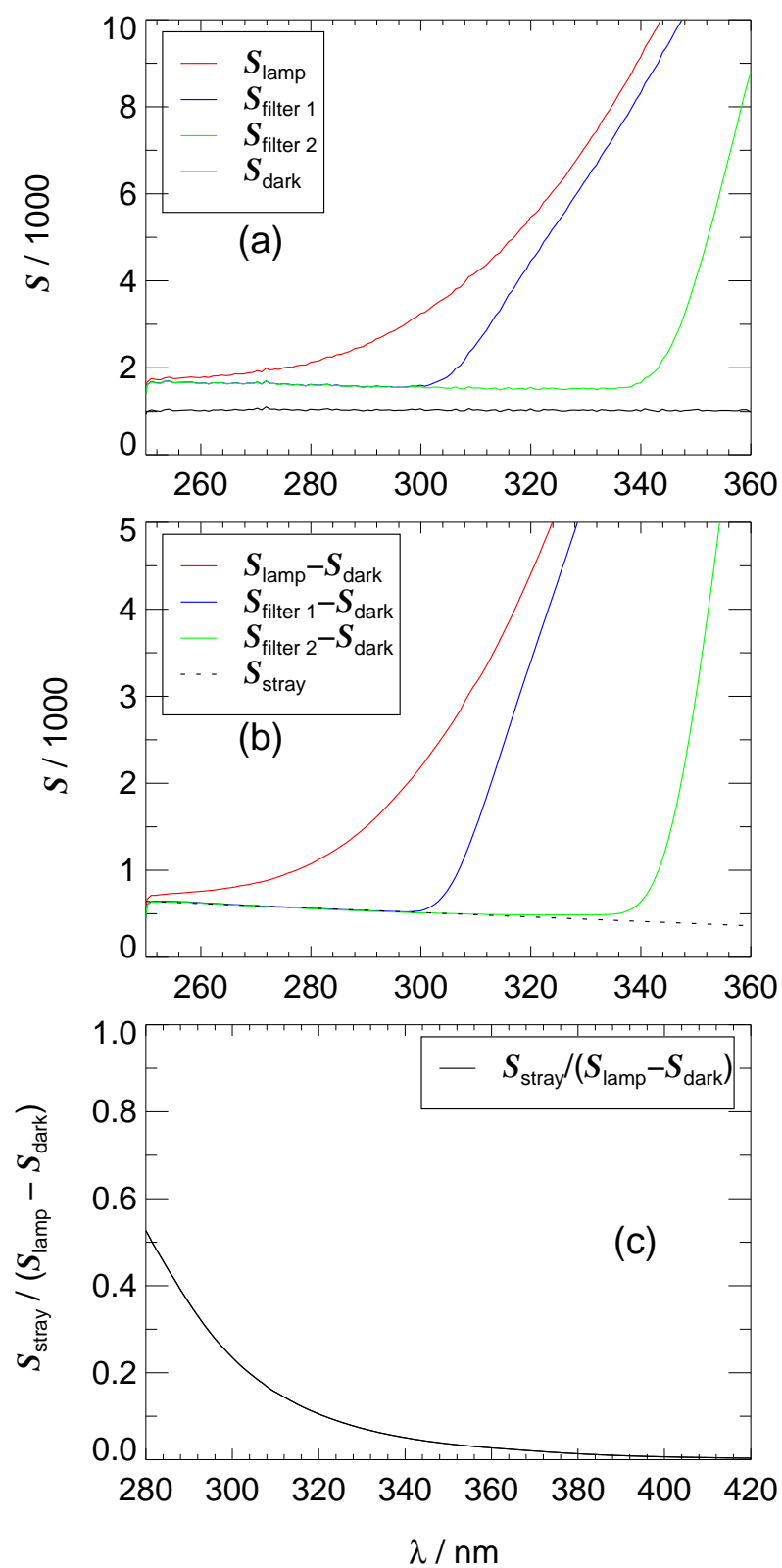

Figure S4: Example signals obtained during laboratory calibration measurements of instrument 85235 with 1000 ms integration time and two cutoff filters. Refer to Sect. 2.2.3 and caption of Fig. 6 for more details. 
Table S1: Downward spectral actinic flux densities $F_{\lambda}$ from radiative transfer calculations for selected wavelengths and solar zenith angles for an altitude of $0 \mathrm{~km}$ and an ozone column of $300 \mathrm{DU}$ (left) and simulated noise equivalent actinic flux densities $F_{\lambda}^{\mathrm{NE}}$ of instrument 62001 for a maximum $300 \mathrm{~ms}$ integration time (right). The entry SZA $>100^{\circ}$ indicates dark conditions. See Tab. 4 for comparison.

\begin{tabular}{|c|c|c|c|c|c|c|c|c|c|c|c|c|c|c|c|c|}
\hline \multirow{2}{*}{$\begin{array}{c}\lambda / \mathrm{nm} \\
\mathrm{SZA} / \mathrm{deg}\end{array}$} & 300 & 350 & 400 & 450 & 500 & 550 & 600 & 650 & 300 & 350 & 400 & 450 & 500 & 550 & 600 & 650 \\
\hline & \multicolumn{10}{|c|}{$F_{\lambda} / 10^{12} \mathrm{~cm}^{-2} \mathrm{~s}^{-1} \mathrm{~nm}^{-1}$} & \multicolumn{6}{|c|}{$F_{\lambda}^{\mathrm{NE}} / 10^{10} \mathrm{~cm}^{-2} \mathrm{~s}^{-1} \mathrm{~nm}^{-1}$} \\
\hline 0 & 2.5 & 190 & 360 & 520 & 510 & 550 & 560 & 550 & 1.6 & 27 & 52 & 54 & 52 & 55 & 59 & 63 \\
\hline 30 & 1.4 & 170 & 340 & 500 & 500 & 540 & 550 & 540 & 1.4 & 26 & 51 & 53 & 51 & 55 & 59 & 62 \\
\hline 50 & 0.31 & 140 & 290 & 450 & 460 & 500 & 520 & 510 & 1.1 & 23 & 47 & 50 & 49 & 53 & 57 & 61 \\
\hline 60 & 0.069 & 110 & 240 & 390 & 410 & 450 & 470 & 480 & 1.0 & 11 & 25 & 46 & 46 & 50 & 54 & 59 \\
\hline 70 & $0.73^{a}$ & 64 & 160 & 280 & 310 & 350 & 370 & 400 & 1.0 & 8.8 & 20 & 40 & 40 & 45 & 49 & 54 \\
\hline 80 & $0.16^{a}$ & 23 & 61 & 110 & 140 & 170 & 180 & 220 & 1.0 & 3.1 & 6.7 & 15 & 16 & 18 & 20 & 23 \\
\hline 84 & $0.095^{a}$ & 12 & 28 & 50 & 60 & 70 & 77 & 100 & 1.0 & 2.2 & 4.7 & 5.3 & 10 & 12 & 13 & 16 \\
\hline 88 & $0.042^{a}$ & 3.3 & 7.4 & 12 & 11 & 9.4 & 7.6 & 13 & 1.0 & 1.3 & 1.4 & 1.5 & 1.4 & 1.3 & 1.3 & 1.8 \\
\hline$>100$ & 0 & 0 & 0 & 0 & 0 & 0 & 0 & 0 & 1.0 & 0.6 & 0.4 & 0.3 & 0.3 & 0.3 & 0.3 & 0.4 \\
\hline
\end{tabular}

${ }^{a} F_{\lambda} / 10^{10} \mathrm{~cm}^{-2} \mathrm{~s}^{-1} \mathrm{~nm}^{-1}$

Table S2: Photolysis frequencies from radiative transfer calculations of downward spectral actinic flux densities for selected solar zenith angles at an altitude of $0 \mathrm{~km}$ and an ozone column of $300 \mathrm{DU}$ (left) and simulated noise equivalent photolysis frequencies of instrument 62001 for a maximum $300 \mathrm{~ms}$ integration time (right). $j\left(\mathrm{O}^{1} \mathrm{D}\right)$ precisions in brackets were obtained by applying variable cutoff wavelengths (Fig. S5). The entry SZA $>100^{\circ}$ indicates dark conditions with zero spectral actinic flux densities. See Tab. 5 for comparison.

\begin{tabular}{ccc|cc}
\hline $\begin{array}{c}\text { SZA } / \\
\text { deg }\end{array}$ & \multicolumn{2}{c}{ photolysis frequency } & \multicolumn{2}{c}{ noise equivalent photolysis frequency } \\
& $j\left(\mathrm{O}^{1} \mathrm{D}\right)$ & $j\left(\mathrm{NO}_{2}\right)$ & $j\left(\mathrm{O}^{1} \mathrm{D}\right)$ & $j\left(\mathrm{NO}_{2}\right)$ \\
\hline 0 & $3.73 \times 10^{-5}$ & $9.00 \times 10^{-3}$ & $1.1 \times 10^{-7}\left(2.5 \times 10^{-8}\right)$ & $1.2 \times 10^{-6}$ \\
30 & $2.72 \times 10^{-5}$ & $8.38 \times 10^{-3}$ & $1.0 \times 10^{-7}\left(2.1 \times 10^{-8}\right)$ & $1.1 \times 10^{-6}$ \\
50 & $1.33 \times 10^{-5}$ & $6.86 \times 10^{-3}$ & $1.0 \times 10^{-7}\left(1.4 \times 10^{-8}\right)$ & $9.6 \times 10^{-7}$ \\
60 & $6.79 \times 10^{-6}$ & $5.46 \times 10^{-3}$ & $1.0 \times 10^{-7}\left(9.6 \times 10^{-9}\right)$ & $7.4 \times 10^{-7}$ \\
70 & $2.37 \times 10^{-6}$ & $3.48 \times 10^{-3}$ & $1.1 \times 10^{-7}\left(5.8 \times 10^{-9}\right)$ & $4.8 \times 10^{-7}$ \\
80 & $4.50 \times 10^{-7}$ & $1.27 \times 10^{-3}$ & $1.1 \times 10^{-7}\left(4.0 \times 10^{-9}\right)$ & $1.7 \times 10^{-7}$ \\
84 & $1.75 \times 10^{-7}$ & $6.14 \times 10^{-4}$ & $1.1 \times 10^{-7}\left(3.6 \times 10^{-9}\right)$ & $9.7 \times 10^{-8}$ \\
88 & $4.13 \times 10^{-8}$ & $1.63 \times 10^{-4}$ & $1.0 \times 10^{-8}\left(2.5 \times 10^{-9}\right)$ & $5.3 \times 10^{-8}$ \\
$>100$ & 0.0 & 0.0 & $1.0 \times 10^{-7}\left(2.5 \times 10^{-9}\right)$ & $2.4 \times 10^{-8}$ \\
\hline
\end{tabular}




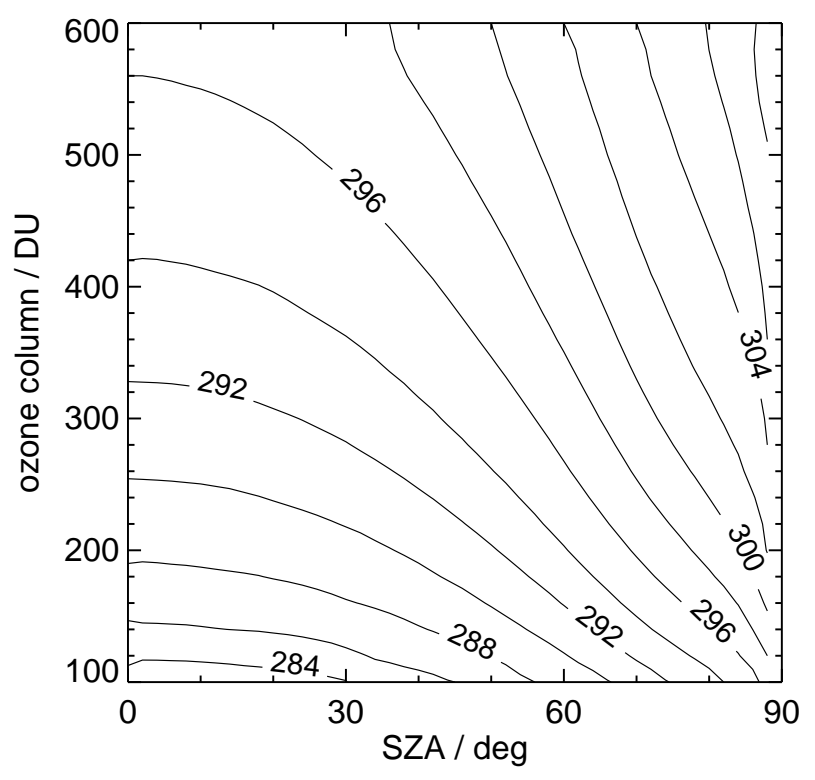

Figure S5: Contour plot of atmospheric cutoff wavelengths (nm) for an altitude of $0 \mathrm{~km}$ as a function of solar zenith angles (SZA) and ozone columns. The data were derived from radiative transfer calculations of downward clear sky spectral actinic flux densities defining a lower limit $F_{\lambda} \leq 5 \times 10^{9} \mathrm{~cm}^{-2} \mathrm{~s}^{-1} \mathrm{~nm}^{-1}$. See Fig. 12 for comparison.

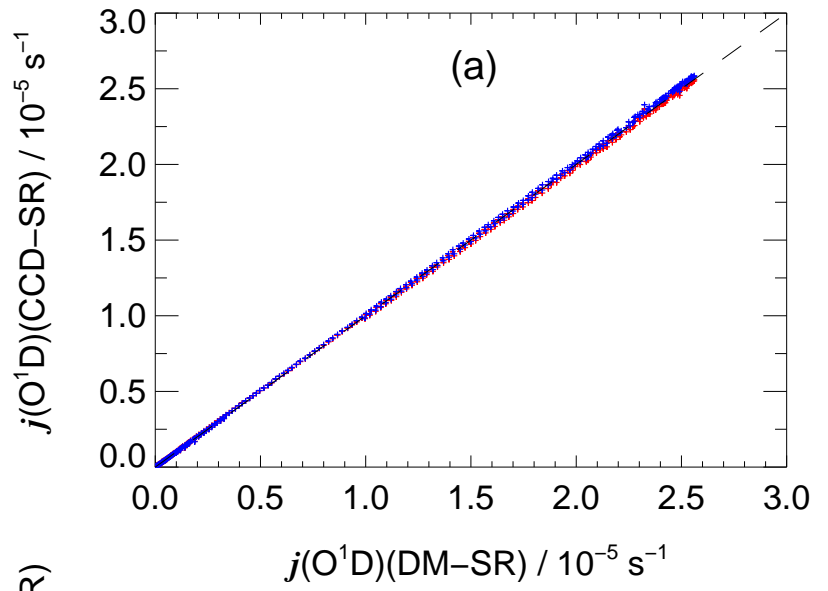

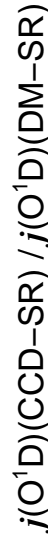
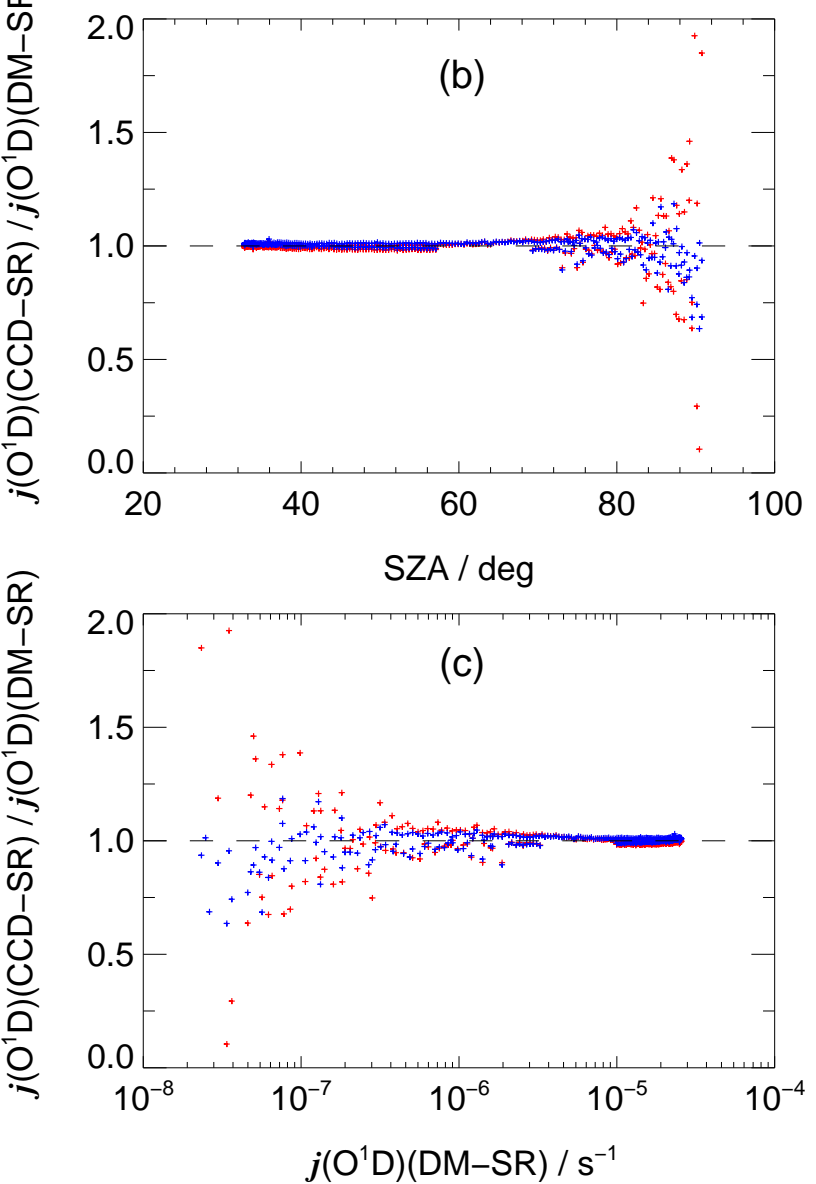

Figure S6: Comparison of $j\left(\mathrm{O}^{1} \mathrm{D}\right)$ photolysis frequencies obtained on the ground with a double-monochromator based reference instrument (DM-SR) and instrument 62001. Measurements were made on 01 Aug 2013 at Jülich (Germany) under clear-sky conditions. Panel (a): correlation plot. Panel (b): ratios as a function of solar zenith angles. Panel (c): ratios as a function of reference values (DM-SR). Dashed lines indicate 1:1 relationships. Scatter in the ratios towards large SZA (middle panels) and low $j\left(\mathrm{O}^{1} \mathrm{D}\right)$ (lower panels) is caused by different detection limits. Blue and red data points were obtained when spectral actinic flux densities below cutoff wavelengths were set to zero and not set to zero, respectively. See Fig. 18 for a typical comparison including more variable, cloudy conditions. 

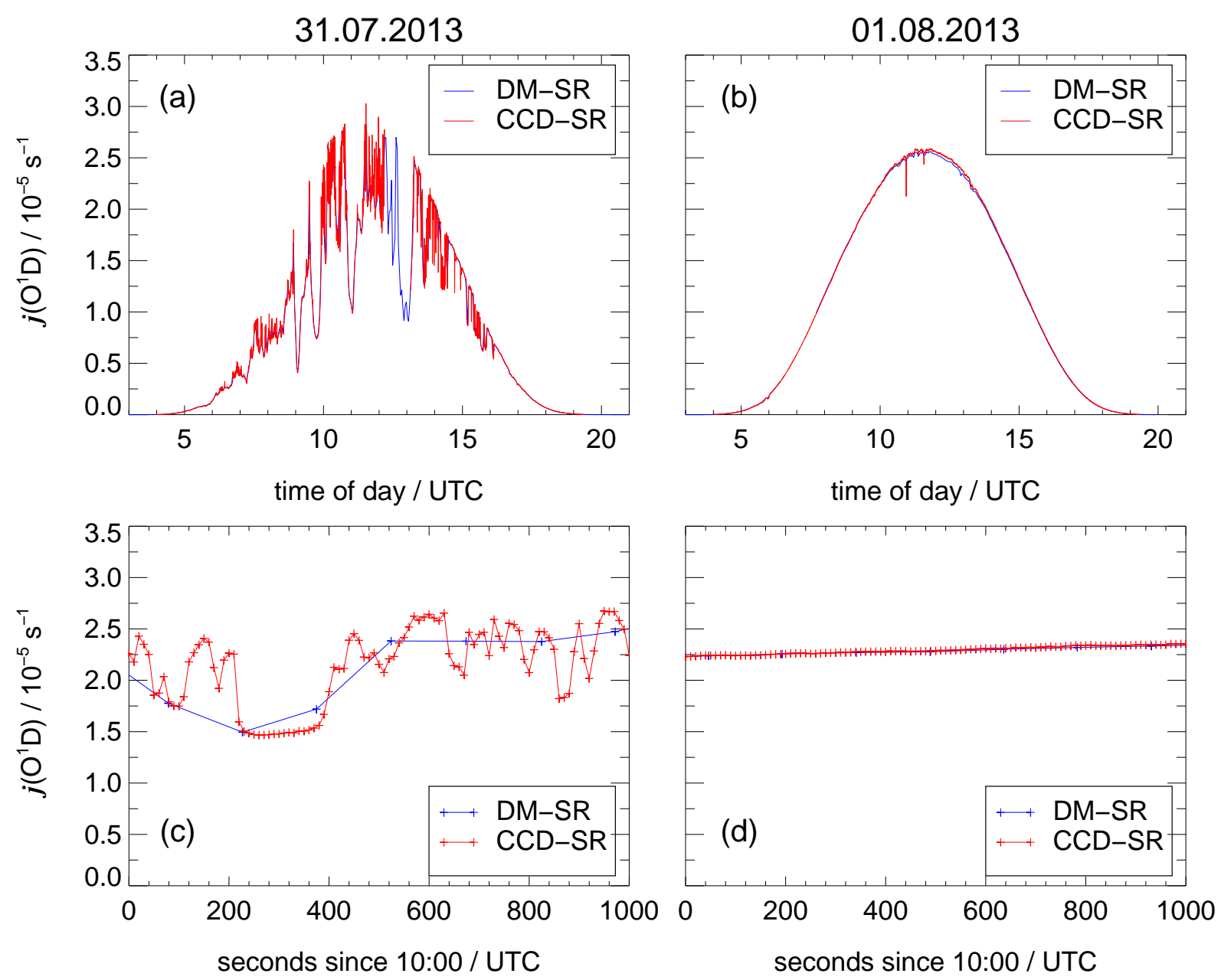

Figure S7: Comparison of $j\left(\mathrm{O}^{1} \mathrm{D}\right)$ photolysis frequencies obtained on the ground at Jülich (Germany) with a double-monochromator based reference instrument (DM-SR) and instrument 62001 (CCD-SR). Measurements of two successive days with broken cloud conditions in panel (a) (31 July) and clear-sky conditions in panel (b) (01 Aug 2013) are shown. Missing data indicate calibration periods for the CCD-SR in panel (a) and the DM-SR in panel (b). The lower panels (c) and (d) show 1000 s periods for each day to demonstrate the effects of different instrument time resolutions ( $\approx 150 \mathrm{~s}$ and $10 \mathrm{~s}$, respectively). There is no synchronization issue on the clear-sky day while for the broken cloud conditions deviations can go in both directions. 